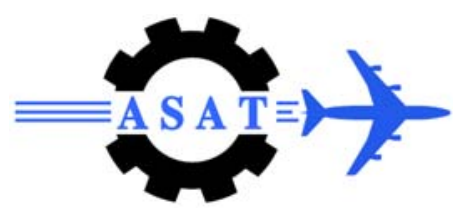

\title{
Investigations of Turbulence Generation in a Model of Solid Rocket Motor Chamber with Sidewall Mass Injection and Endwall Disturbance
}

\author{
S. M. El-Behery ${ }^{*}$, A. M. Hegab ${ }^{\dagger}$, A. E. Balabel ${ }^{*}$, M. N. Nasr ${ }^{*}$
}

\begin{abstract}
The present paper presents numerical investigations of the internal flow field in a nozzleless solid rocket motor chamber with compressible transpiration and endwall disturbance. More attention is given for predicting the induced turbulent flow inside the chamber. The considered physical configuration of the nozzleless solid rocket motor consists of a two-dimensional rectangular and porous chamber fitted at the head end with a specifiedfrequency-oscillating piston and it is opened to atmospheric plenum in the downstream direction. The numerical study is performed by solving the unsteady RANS equations along with the energy equation using the control volume approach on the basis of a staggered grid system. Different two-equation turbulence models have been implemented and their accuracy is estimated through a comparison with the available experimental measurements. A fairly general agreement has been obtained between the numerical and the experimental results. Moreover, the obtained results showed that complex turbulent internal flow patterns are induced inside the chamber due to the strong interaction of the sidewall injection with the traveling acoustic waves. Such complex internal structure is shown to be dependent on piston frequency and sidewall mass flux.
\end{abstract}

Keywords: Compressible unsteady flow, endwall disturbance, permeable Walls, solid rocket motor chamber, turbulence models.

\section{Nomenclature}

$b_{n}$ wave number

$\mathrm{C}_{\mathrm{o}}^{\prime}$ speed of sound, $\mathrm{m} / \mathrm{s}$

$\mathrm{C}_{\mathrm{v}}$ specific heat at constant volume

$\mathrm{C}_{\mathrm{p}}$ specific heat at constant pressure

$\mathrm{E}_{\mathrm{t}}$ total energy

$\mathrm{H}^{\prime}$ channel half-height, $\mathrm{cm}$

$\mathrm{H}$ dimensionless channel half-height

$\mathrm{K}$ thermal conductivity

L' channel length, cm

L dimensionless channel length

M Mach number

$\mathrm{n}$ wave number index

$\mathrm{P}$ static pressure

$\mathrm{P}_{\mathrm{atm}}$ atmospheric pressure

$\mathrm{P}^{\prime}{ }_{\mathrm{o}}$ stagnation pressure, $\mathrm{Pa}$
$\tilde{P}$ dimensionless pressure perturbation

Pr Prandtl number

Re Reynolds Number

$\mathrm{T}$ temperature

$\mathrm{T}_{\mathrm{o}}^{\prime}$ stagnation temperature, $\mathrm{K}$

$\mathrm{t}$ time

$\mathrm{t}_{\mathrm{a}}^{\prime}$ acoustic time, $\mathrm{L}^{\prime} / \mathrm{C}_{\mathrm{o}}^{\prime}$

$\mathrm{U}^{\prime}{ }_{\mathrm{o}}$ reference axial speed, $\mathrm{m} / \mathrm{s}$

$\mathrm{u}$ axial speed

$\mathrm{V}$ transverse speed

$V^{\prime}$ inj reference injection speed, $\mathrm{m} / \mathrm{s}$

$\mathrm{x}$ axial coordinate

$\mathrm{X}^{\prime}$ dimension axial coordinate

$\mathrm{y}$ transverse coordinate

\footnotetext{
* Faculty of Engineering, Menoufiya University, Shebin El-Kom, Egypt

${ }^{\dagger}$ Professor, Corresponding Author, Faculty of Engineering, Menoufiya University, Shebin

El-Kom, Egypt, Hegab2002us@yahoo.com
} 


\section{Greek symbols \\ $\delta$ aspect ratio \\ $\varepsilon$ transient axial velocity amplitude \\ $\gamma$ ratio of specific heat \\ $\mu^{\prime}$ dynamic viscosity, pa.s}

$\begin{array}{ll}\Omega & \text { vorticity } \\ \omega & \text { frequency } \\ \rho & \text { density } \\ \rho_{\mathrm{o}}^{\prime} & \text { stagnation density, } \mathrm{Kg} / \mathrm{m}^{3}\end{array}$

inj injection

\section{Introduction}

Flow through porous channels with sidewall injection and endwall disturbance is used to mimic the unsteady mass addition due to irregular propellant burning and fluid dynamics in a solid rocket motor chamber (SRMC) [1]. The prediction of such complex flow can provide the rocket's designers with the essential data required for the optimum rocket design and operation. Consequently, such complex fluid dynamics problem has received a great attention over the last decade [2]. In such configuration, the internal flow exhibits time-dependent, compressible, laminar-turbulent transition and turbulent characteristics over a large portion of the SRMC [3].

The recent studies of our research group were directed to simulate the internal fluid dynamics inside SRMC with or without nozzle configuration [4]. In such context, different turbulence models have been assessed and their accuracy was evaluated through an extensive comparison with the previous experimental data. It was found that, among different turbulence models adopted, the standard k- $\varepsilon$ model could fairly accurately predict the flow characteristics with moderate computational cost. However, this turbulence model could not be suitable in simulation of nozzle configuration as it can be seen in our recent study [5]. Therefore, in the present study two different, however, based k- $\varepsilon$ models are implemented; namely, the standard two-equation k- $\varepsilon$ turbulence model [6] (ST-TM) and its modified version developed by Chen and Kim [7] (CK-TM).

Although such problem has been investigated using Large Eddy Simulation technique (LES) [8], however, the computational demand of LES and the complexity encountered gave the superiority to the two-equation based k- $\varepsilon$ turbulence model, especially when the differences in the predicted average quantities were not large [9].

Most of the previous investigations have demonstrated that unsteady sidewall mass injection in SRMC is the immediate source of acoustic disturbances that propagate in the low axial Mach number (M), high Reynolds number (Re) mean flow [10]. The analytical results provided therein have proved that an interaction between the acoustic transients and the fluid injected from the sidewall causing transverse axial velocity gradients (vorticity) and transverse temperature gradients (heat transfer) to appear on the sidewalls. The prior as well as accurate assumption of the injected flow could simulate the corrugation that arises from the burning of composite propellants.

As an alternative to that, acoustics in SRMC can be generated by a vibrating piston at the endwall boundary. In such cases, the opposite boundary conditions in the SRMC play a significant role in the behaviour of the internal fluid dynamics as well as the acoustic 
characteristics, for more details one can see [11, 12]. Moreover, this problem has been investigated both analytically and experimentally by our research group [13], and the results showed that, the geometry of the variable area parts connected to the SRMC had a significant effect on the generated complex wave pattern inside the chamber. However, the effect of turbulence transition inside the SRMC was not considered.

As a result, in the present study, the effect of the generated endwall disturbance on the internal flow in SRMC with sidewall mass injection is investigated numerically considering the turbulence characteristics of the induced flow. The numerical results were obtained using a FORTRAN computer program developed by our numerical group team. This program has been constructed, validated against a wide range of Computational Fluid Dynamics problems in different aerodynamics applications with reacting flow. Further development of the present work is currently under investigation through our research group by including the additional effects of either divergent or convergent-divergent portions on the acoustic wave deformation when these variable area geometries are connected to the open end of the SRMC.

The rest of the paper is organized as follows. In section 2, the RANS equations for timedependent, compressible turbulent flows along with the implemented turbulence models are presented. Following, in section 3, the numerical procedure and the associated boundary conditions are explored. In section 4, the obtained numerical results are compared with both analytical and published experimental data and discussed in detail. Finally, in section 5, conclusions of the present work are drawn.

\section{Mathematical Formulation}

The governing conservation equations of the flow under consideration can be written for timedependent, compressible and turbulent flow as follows:

\section{Continuity Equation:}

$$
\frac{\partial \rho}{\partial t}+\frac{\partial}{\partial x_{i}}\left(\rho u_{i}\right)=0
$$

\section{Momentum Equation:}

$$
\frac{\partial\left(\rho u_{i}\right)}{\partial t}+\frac{\partial}{\partial x_{j}}\left(\rho u_{i} u_{j}\right)=-\frac{\partial p}{\partial x_{i}}+\frac{\partial}{\partial x_{j}}\left[\mu\left(\frac{\partial u_{i}}{\partial x_{j}}+\frac{\partial u_{j}}{\partial x_{i}}-\frac{2}{3} \delta_{i j} \frac{\partial u_{l}}{\partial x_{l}}\right)\right]+\frac{\partial}{\partial x_{j}}\left(-\rho \overline{u_{i}^{\prime} u_{j}^{\prime}}\right)
$$

\section{Energy Equation:}

$$
\frac{\partial(\rho e)}{\partial t}+\frac{\partial\left[u_{j}(\rho e+p)\right]}{\partial x_{j}}=\frac{\partial}{\partial x_{j}}\left[\left(\lambda+C_{p} \mu_{t} / \operatorname{Pr}_{t}\right) \frac{\partial T}{\partial x_{j}}-u_{i}\left(\rho \overline{u_{i}^{\prime} u_{j}^{\prime}}\right)\right]
$$

where, $u_{i}$ denotes the mean velocities and $u_{i}^{\prime}$ and $u_{j}^{\prime}$ are the turbulent fluctuations, $\rho$ is the density, $p$ is the pressure, $\lambda$ is the thermal conductivity, $C_{p}$ is the specific heat at constant pressure, $\operatorname{Pr}_{t}$ is the turbulent Prandtl number and $\mu$ is the dynamic viscosity. The total energy 
per unit volume is defined as: $\rho e=\rho C_{v} T+0.5 \rho u_{i}{ }^{2}$, where $C_{v}$ is the specific heat at constant volume and $T$ is the temperature.

The momentum equations contain additional terms, known as Reynolds stresses, that represent the effects of turbulence. These Reynolds stresses, $-\rho \overline{u_{i}^{\prime} u_{j}^{\prime}}$, must be modeled in order to close governing system of euqtions. That can be done through the approperiate choice of the turbulence model.

For compressible flow, equations 1, 2 and 3 can be interpreted as Favre-averaged conservation equations, with the velocities representing mass-averaged values. The Reynoldsaveraged conservation equations for time-dependent compressible turbulent flow along with a turbulence model are coupled with the equation of state, $p=\rho R T$ to close the system of governing equations.

\section{Turbulence modeling}

In industrial CFD applications, RANS modeling remains one of the main approaches when dealing with turbulent flows. The Reynolds-averaged approach to turbulence modeling requires appropriate modeling of the Reynolds stresses in Eq.2. The common method to relate the Reynolds stresses to the mean velocity gradients is based on Boussinesq hypothesis [14]:

$$
-\rho{u_{i}^{\prime}}_{i}^{\prime}{ }_{j}=\mu_{t}\left(\frac{\partial u_{i}}{\partial x_{j}}+\frac{\partial u_{j}}{\partial x_{i}}\right)-\frac{2}{3}\left(\rho k+\mu_{t} \frac{\partial u_{k}}{\partial x_{k}}\right) \delta_{i j}
$$

where, $\delta_{i j}$ is the Kronecker delta function $\left(\delta_{i j}=1\right.$ if $\mathrm{i}=\mathrm{j}$ and $\delta_{i j}=0$ if $\mathrm{i} \neq \mathrm{j}$ ), $k$ is the turbulent kinetic energy. One of the most challenging problem of turbulent flow is the estimation of the turbulent viscosity, which, unlike the dynamic viscosity, not a flow property.

For the calculation of the turbulent characteristics, both the standard (ST-TM) and the extended $k-\varepsilon$ turbulence model (CK-TM) are applied, where the transport equation of the turbulent kinetic energy and its dissipation rate can be written as follows [7]:

$$
\begin{gathered}
\frac{\partial(\rho k)}{\partial t}+\frac{\partial}{\partial x_{j}}\left(\rho u_{j} k\right)=\frac{\partial}{\partial x_{j}}\left[\left(\mu+\frac{\mu_{t}}{\sigma_{k}}\right) \frac{\partial k}{\partial x_{j}}\right]+\rho\left(P_{r}-\varepsilon\right) \\
\frac{\partial(\rho \varepsilon)}{\partial t}+\frac{\partial}{\partial x_{j}}\left(\rho u_{j} \varepsilon\right)=\frac{\partial}{\partial x_{j}}\left[\left(\mu+\frac{\mu_{t}}{\sigma_{\varepsilon}}\right) \frac{\partial \varepsilon}{\partial x_{j}}\right]+\rho\left(C_{1 \varepsilon} P_{r} \frac{\varepsilon}{k}-C_{2 \varepsilon} \frac{\varepsilon^{2}}{k}+C_{3 \varepsilon} \frac{P_{r}^{2}}{k}\right)
\end{gathered}
$$

where $P_{r}$ and $\varepsilon$ represent the production rate and the dissipation rate of the turbulent kinetic energy, respectively, The production rate is related to the mean strain of the velocity field through the Boussinesq assumption. That is,

where $S$ is defined as:

$$
P_{r}=\mu_{t} S^{2}
$$




$$
S=\sqrt{\frac{1}{\rho}\left(\frac{\partial u_{j}}{\partial x_{i}}+\frac{\partial u_{i}}{\partial x_{j}}-\frac{2}{3} \delta_{i j} \frac{\partial u_{k}}{\partial x_{k}}\right) \frac{\partial u_{i}}{\partial x_{j}}}
$$

The turbulent viscosity is given by:

$$
\mu_{t}=\rho C_{\mu} \frac{k^{2}}{\varepsilon}
$$

The constants of the adopted turbulence models are given as follows [6, 7]:

\begin{tabular}{|c|c|c|c|c|c|c|}
\hline $\begin{array}{c}\text { Turbulence } \\
\text { model }\end{array}$ & $\sigma_{k}$ & $\sigma_{\varepsilon}$ & $C_{1 \varepsilon}$ & $C_{2 \varepsilon}$ & $C_{3 \varepsilon}$ & $C_{\mu}$ \\
\hline ST-TM & 1.0 & 1.3 & 1.44 & 1.92 & 0.0 & 0.09 \\
\hline CK-TM & 0.75 & 1.15 & 1.15 & 1.9 & 0.25 & 0.09 \\
\hline
\end{tabular}

\section{Numerical Procedure}

The above system of equations, is solved by the numerical method proposed by Patankar [15] based on the control volume approach. The governing equations are discretized using hybrid scheme for all variables except the density which is interpolated using first order upwind scheme in order to obtain a linear system of algebraic equations. This system of equations is solved using the TDMA described in [15]. Second order implicit discretization is applied to the unsteady term. An important step of the above numerical procedure is the linearization of the source terms that vary according to the equations considered. The solution is carried out using SIMPLE algorithm which is extended to compressible flow according to [16] and the program is considered to reach the final solution when the maximum normalized residual approaches a value of $10^{-4}$ during each time step. The time interval $\Delta \mathrm{t}$ is assumed to be constant and small enough to achieve a stable and non-oscillatory solution.

\subsection{Boundary Conditions}

There are three kinds of boundary conditions used in this study, see for more detail Fig. 1. The first case in which the air is injected uniformly across the sidewalls of the channel and the head end is closed while the other free end is open to atmosphere. At the head end, $x=0$ the no slip boundary conditions are applied, $\mathrm{u}=\mathrm{v}=0$, while at the duct exit, the pressure is taken as an atmospheric pressure and the other parameters are extrapolated using zero gradient at exit. At the sidewalls, the no slip condition is applied for the stream-wise component of velocity, but the crosswise velocity is taken to be the injection velocity $v_{\text {inj }}$.

The second case of the boundary conditions is concerned with studying the internal flow field when only acoustic waves are generated at the closed end while the other free end is open to atmosphere. The same boundary conditions for the case (1) are applied except that; the no slip condition is applied also to the velocity components at sidewalls, i.e. $\mathrm{u}=\mathrm{v}=0$. At the head end, $\mathrm{x}=0$, the stream-wise velocity is considered in the form $\mathrm{u}=\varepsilon \sin (\omega \mathrm{t})$, where $\varepsilon$ and $\omega$ represent the wave amplitude and the forced frequency, respectively.

In the third case of boundary conditions, the air is injected uniformly across the sidewall of the chamber and acoustic waves are generated at the head end while the other free end is open to atmosphere. The same boundary conditions for the case (1) are applied except that; at the head end $(\mathrm{x}=0), \mathrm{u}=\varepsilon \sin (\omega \mathrm{t})$. At the sidewalls, the crosswise velocity is taken to be the injection velocity $\mathrm{v}_{\text {inj. }}$. 
In the following sections, samples of the obtained results are illustrated. The key elements of the performed cases are the injection velocity $V_{\text {inj }}$ and the axial perturbation velocity of the vibrated piston $\mathrm{u}_{\mathrm{p}}$. Three different cases are considered as follows:

\begin{tabular}{|l|c|c|}
\hline & $\mathrm{V}_{\mathrm{inj}}$ & $\mathrm{u}_{\mathrm{p}}$ \\
\hline CASE I & 0.0 & $u=\varepsilon \sin (\omega t)$ \\
\hline CASE II & $\left|v_{i n j}\right|=0.1273 \mathrm{~m} / \mathrm{sec}$ & 0.0 \\
\hline CASE III & $\left|v_{i n j}\right|=0.1273 \mathrm{~m} / \mathrm{sec}$ & $u=\varepsilon \sin (\omega t)$ \\
\hline
\end{tabular}

where $\varepsilon=\omega R=35.91 \mathrm{~m} / \mathrm{sec}$, and $\omega=\frac{2 \pi n}{60}=378.04 \mathrm{rad} / \mathrm{sec}$. The first case is performed both numerically and anlytically and the results from both are compared together. In the second case, the numerical results obtained from the two implemented turbulence models are compared with the experimental measurements of [17]. In the third case, the numerical results obtained from laminar calculation are compared with the turbulent flow results obtained from the second turbulence model.

\subsection{Analytical Solution}

In the present section, an analytical solution is developed for the reduced form of the governing system of equations. The analytical results obtained give an important insight into the preliminary results of the problem under consideration and it might be used as an indication for the logical level of both numerical and experimental results.

The analytical approach is based on the reduced form of the Navier-Stokes equations using asymptotic techniques [18-20] and the final solution for pressure and velocity can be written as follows:

The non resonance acoustic solution for axial velocity is given by:

$$
\bar{u}(\bar{x}, \bar{t})=\bar{\varepsilon} \sin (\bar{\omega} \bar{t})+\sum_{n=0}^{\infty} 2 \frac{\bar{\varepsilon} \bar{\omega}}{b_{n}^{2}-\bar{\omega}^{2}}\left(\frac{\bar{\omega}}{b_{n}^{2}} \sin (\bar{\omega} \bar{t})-\sin \left(b_{n} \bar{t}\right)\right) \sin \left(b_{n} \bar{x}\right) \text { for } \bar{\omega} \neq \mathrm{b}_{\mathrm{n}^{*}}
$$

where $b_{n}$ is the wave number, $b_{n}=(n+1 / 2) \pi$, and $\bar{\omega}$ is the dimensionless forced frequency.

The perturbation pressure $\bar{p}(\bar{x}, \bar{t})$ is found using the following equations [19]:

$$
\frac{\partial \bar{u}(\bar{x}, \bar{t})}{\partial \bar{t}}=-\frac{1}{\gamma M} \frac{\partial \bar{p}(\bar{x}, \bar{t})}{\partial \bar{x}}
$$

The boundary condition at the duct head end, $\bar{x}=1$ reads $\bar{p}=1$. Integrating Eq.11 yields the pressure perturbation in the form:

$$
\begin{aligned}
& \tilde{p}(\bar{x}, \bar{t})=1.0+\gamma M \bar{\varepsilon} \bar{\omega}(1.0-\bar{x}) \cos (\bar{\omega} \bar{t})+ \\
& \gamma M \sum_{n=0}^{\infty} \frac{2 \bar{\varepsilon} \bar{\omega}}{b_{n}^{2}-\bar{\omega}^{2}}\left(\frac{\bar{\omega}^{2}}{b_{n}^{2}} \sin (\bar{\omega} \bar{t})-b_{n} \sin \left(b_{n} \bar{t}\right)\right) \frac{\cos \left(b_{n} \bar{x}\right)}{b_{n}}
\end{aligned}
$$


In the above equations the overbar means dimensionless quantity. The following parameters are used for non-dimensionalization:

$$
\bar{u}=u / U_{z o}, \bar{\varepsilon}=\varepsilon / U_{z o}, \bar{\omega}=\omega L / C_{z o}, \bar{t}=t C_{z o} / L, \bar{x}=x / L, \bar{p}=p / p_{o}
$$

where $C_{z o}=\left(\gamma R T_{o}\right)^{0.5}$ is the charachteristic sound velocity, $U_{z o}=M . C_{z o}$ is the charachteristic axial velocity and $p_{o}$ is the pressure at duct outlet.

\section{Results and Discussions}

The investigation of the flow field inside the chamber of the solid rocket motor chamber is employed. The current study emphasizes the acoustic fluid dynamics interaction mechanism and the accompanying unsteady rotational fields. Moreover, the effect of generated turbulence on these unsteady rotational fields is considered for the first time according to our background. Several cases are performed in order to validate the developed numerical codes.

\subsection{Case I}

The first set of the results are devoted to compare the numerical solution with the analytical one for pure acoustic flow field inside SRMC with endwall disturbances only. The following results, shown in figure 1, illustrate a comparison between the numerical results and the analytical analysis for this case, at different axial location inside the SRMC. The comparisons showed a fairly reasonable agreement between the numerical and analytical results for both the axial velocity and the pressure time histories. However, an important feature of the numerical results for the axial velocity profile at $\mathrm{x} / \mathrm{L}=0.76$, is the appearance of higher harmonics oscillations which represent the eigenfunction mode contributions at different time intervals. This interesting phenomenon can be attributed to the nonlinear terms included in the numerical simulation and neglected in the analytical analysis. The important of including the nonlinear terms is clearly visible in the pressure profiles obtained from the numerical results and the analytical analysis, where discrepancies can be observed. Furthermore, it can be seen that, the amplitude of the numerically predicted pressure is consistent with the analytical analysis in such a manner, the oscillation decreases as the axial distance increases downstream. This behaviour may be attributed to the wave attenuation due to the boundary layer development on the duct sidewalls and acoustic streaming. The important of such comparisons is to indicate the ability of the developed numerical code to predict the full features of the generated acoustic field that consist of the fundamental and higher harmonics inside the interior SRMC.

\subsection{Case II}

In the present case a further validation of the obtained numerical results for another case is carried out by comparing the results with the experimental measurements of [17]. The computational domain is shown in figure 2, where a sidewall mass is injected from the lower wall with a rigid wall on the opposed direction. The chamber is closed from the endwall and opened to the atmospheric pressure on the other side. The current comparison could, to some extent, assist the accuracy of the turbulence models adopted. 

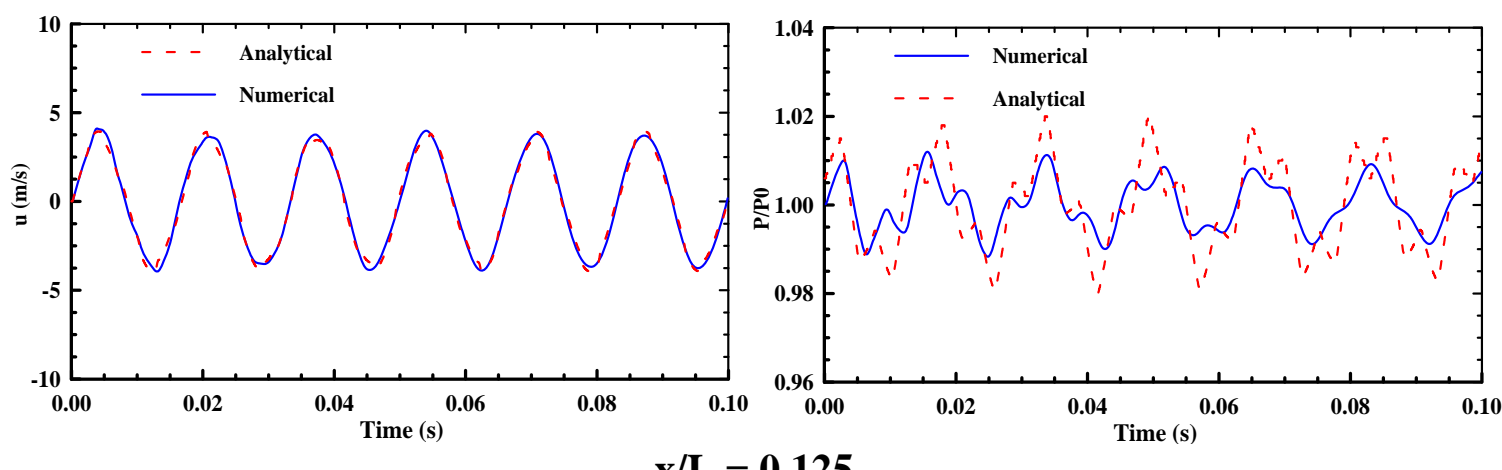

$\mathrm{x} / \mathrm{L}=\mathbf{0 . 1 2 5}$
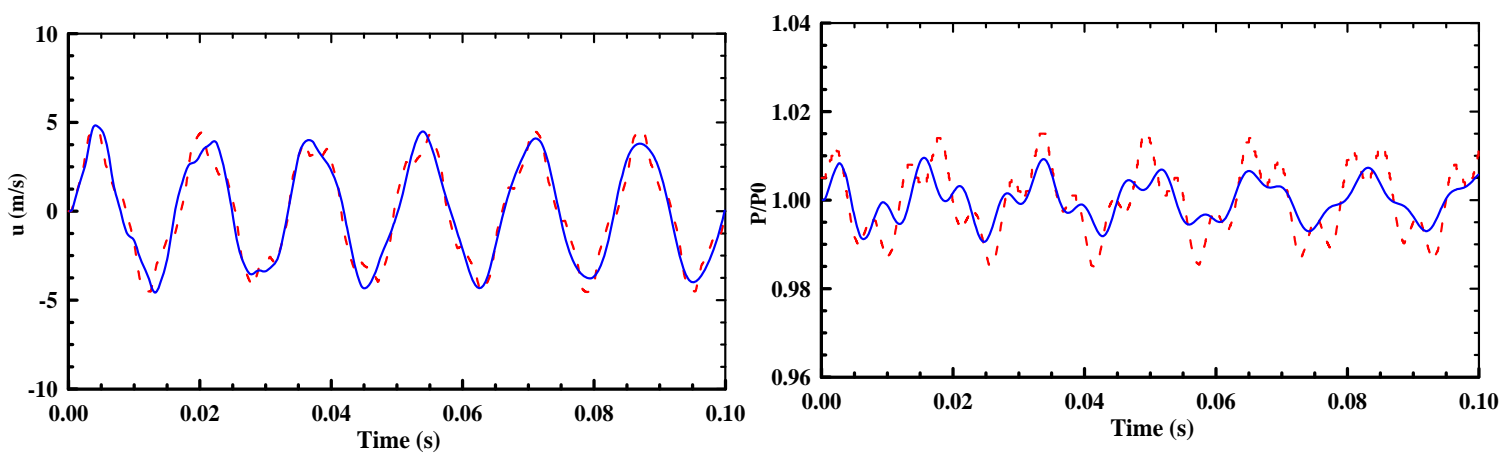

$\mathrm{x} / \mathrm{L}=\mathbf{0 . 5 5 7}$
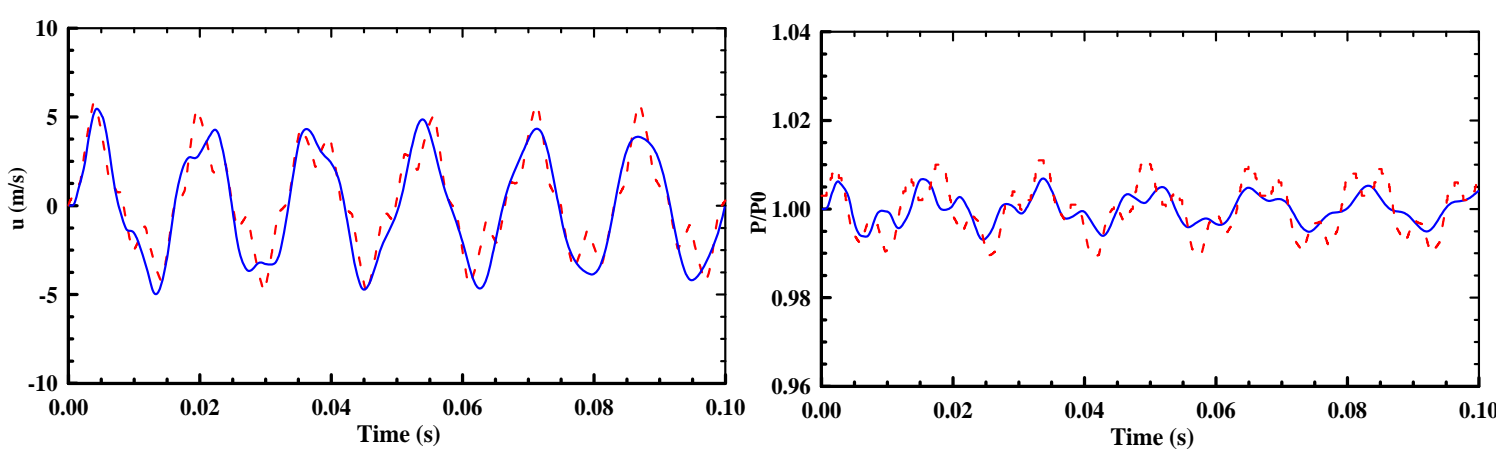

$\mathrm{x} / \mathrm{L}=\mathbf{0 . 7 6}$

Figure 1. Comparison of axial velocity and pressure time histories for CASE I, (numerical and analytical comparison).

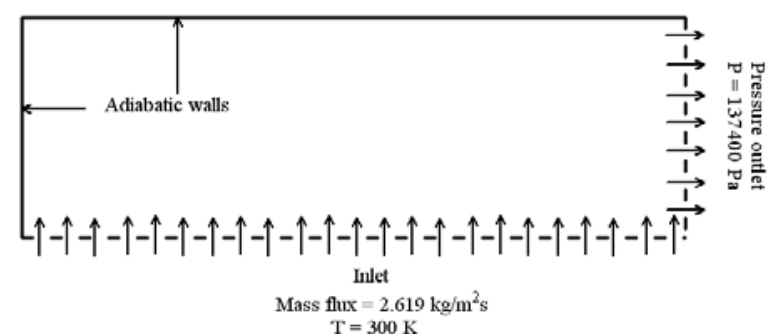

Figure 2. SRMC with porous wall at lower side 

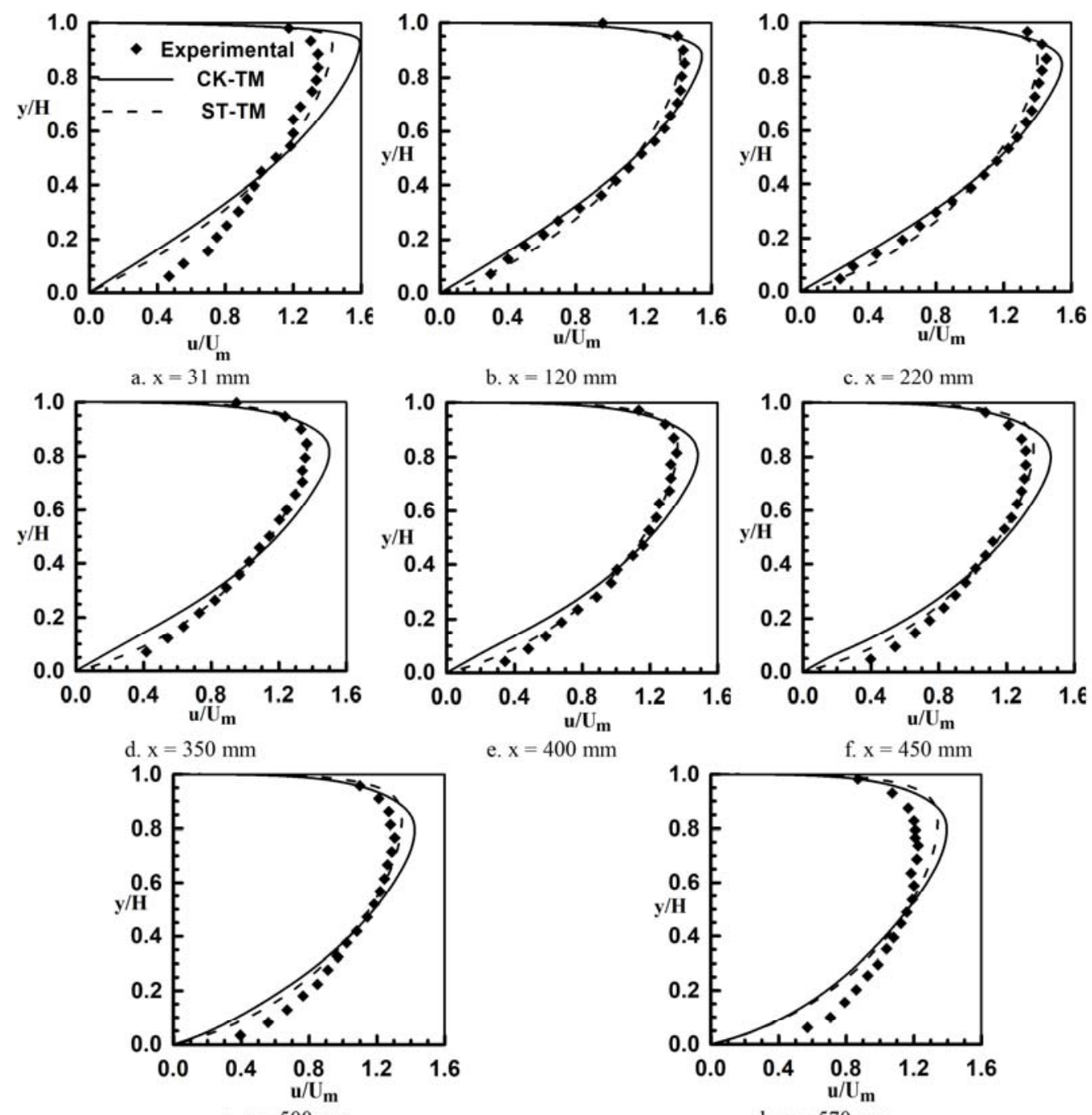

e. $x=400 \mathrm{~mm}$

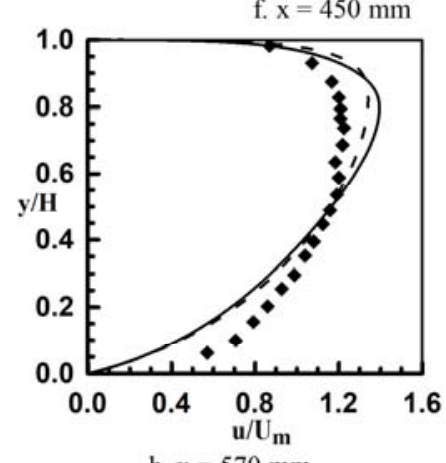

g. $\mathrm{x}=500 \mathrm{~mm}$

h. $x=570 \mathrm{~mm}$

Figure 3-a. Comparison of axial velocity and pressure time histories for CASE II, (numerical and experimental comparison).

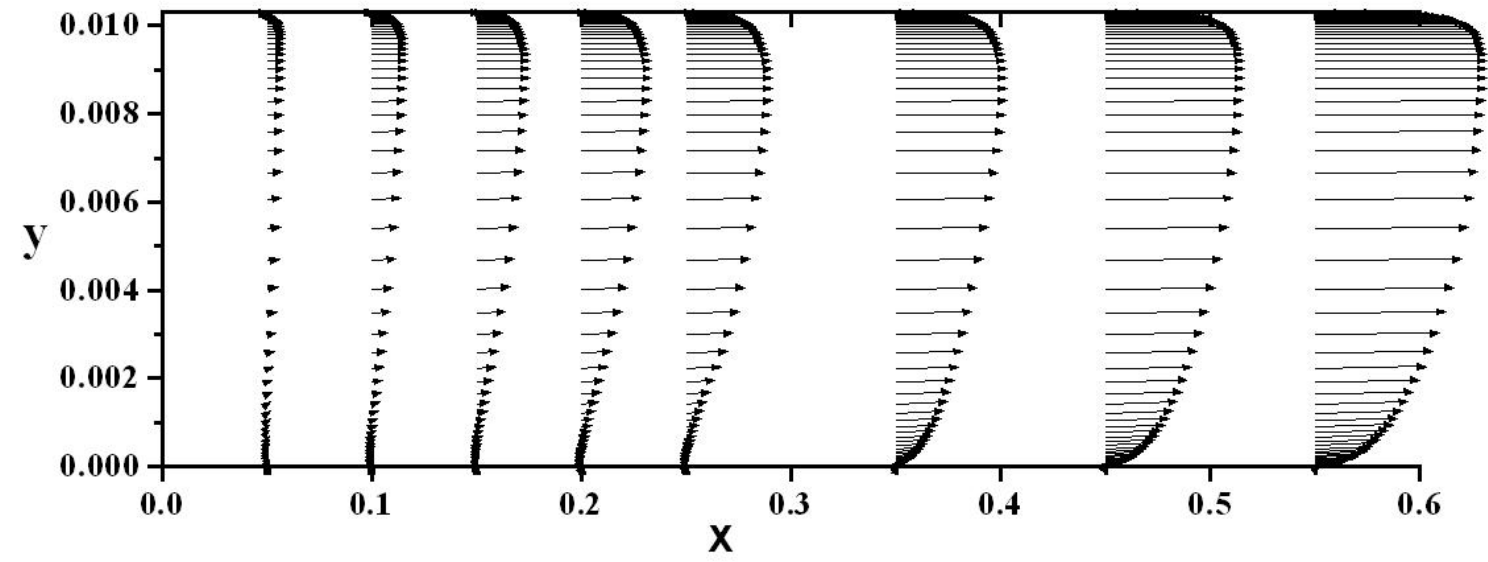

Figure 3-b. Vector plot of the internal flow field of SRMC with sidewall injection 


\subsubsection{Mean dimensionless velocity profile at different axial locations}

The mean velocity and the Reynolds-stress profiles are compared with the experimental measurements of [17], at different axial location; $x=31 ; 120 ; 220 ; 350 ; 400 ; 450 ; 500$ and $570 \mathrm{~mm}$. This is related to the chamber half hight $H$ according to the dimensionless distances: $x / H=3 ; 11.65 ; 21.36 ; 33.98 ; 38.83 ; 43.69 ; 48.54$ and 55.34 , respectively. Figure 3 -a shows the mean streamwise velocity profiles normalized by the local mean streamwise velocity $U_{m}$, where $U_{m}=\frac{1}{H} \int_{0}^{H} u d y$.

The numerical results obtained from the two adopted turbulence models are compared with the experimental data in global coordinates $y / H$. As noticed, the velocity increases rapidly in the boundary layer generated on the solid wall compared with that on the porous wall, as shown in figure 3-b, where the vector plot of the internal velocity filed is illustrated.

Both turbulence models applied could qualitatively predict the velocity profiles at all other locations. However, it is noticed that the standard $k-\varepsilon$ ST-TM turbulence model and its extended form of Chen and Kim CK-TM represented only the core flow and clear deviations are observed near the two wall in spite of the absence of adverse pressure gradient in the present simulation. The failure of the turbulence models used near wall is not caused by the near-wall treatment, but can be produced by the isotropic assumptions in the turbulence model itself.

\subsubsection{Streamwise root-mean-square (RMS) intensity at different axial locations}

Figure 4 shows the streamwise root-mean-square (RMS) turbulence intensity $u^{\prime}$ normalized by the local bulk streamwise velocity $U_{m}$ at four axial locations reading: $x=220 ; 350 ; 450$ and $570 \mathrm{~mm}$, respectively. It is apparent that there are always peaks in the profiles near the porous wall surface. That is clearly visible in the experimental and numerical data. The known near-wall stress peaks at the other solid wall due to the boundary layer development and the strong near-wall velocity gradient couldn't be experimentally captured, while they are numerically observed in the turbulence models applied. Except the first location, that is at near of the transition from laminar to turbulent flow, the two turbulence models adopted present qualitatively good agreement with the experimental data. The higher levels observed in the near-wall turbulence intensity are probably due to the pseudo-turbulence of the injected flow. However, all computations predict somewhat higher values of turbulence intensity compared with the experimental data.

\subsubsection{Turbulent shear stress at different axial locations}

The turbulent shear stress component $u^{\prime} v^{\prime} / U_{m}^{2}$ is illustrated in Fig. 5. A general fairly qualitatively agreement is obtained according to the simple turbulence models adopted. The failure of the turbulence models adopted in prediction the turbulent shear stress can be referred to the assumption of Boussinesq relation in describing the Reynolds stresses. Recent investigations have showed that, the implementation of a nonlinear constitutive relation in describing the Rynolds stresses, or in other words, the application of the so-called nonlinear turbulence models, could improve the prediction of Reynolds stresses in such complex flow. This problem was fully discussed in [21]. 


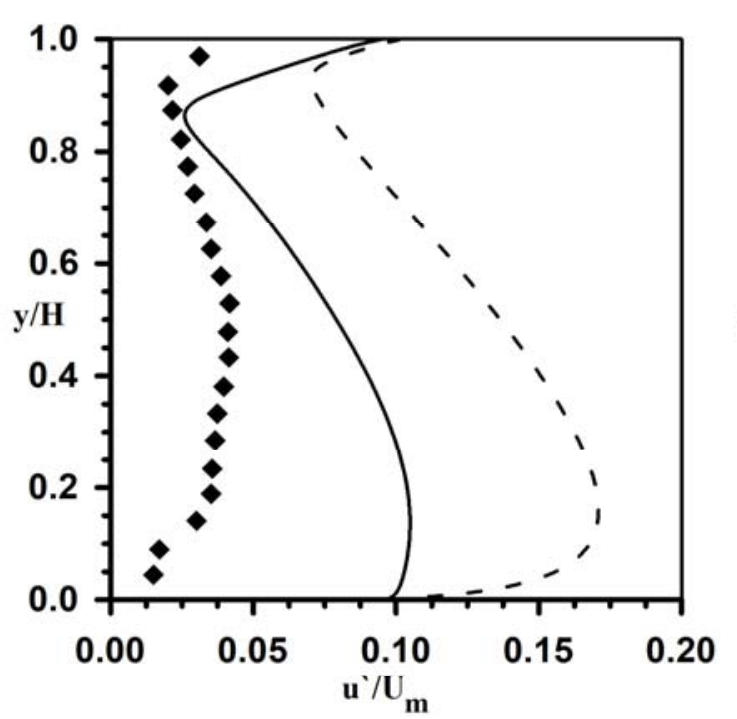

a. $x=220 \mathrm{~mm}$

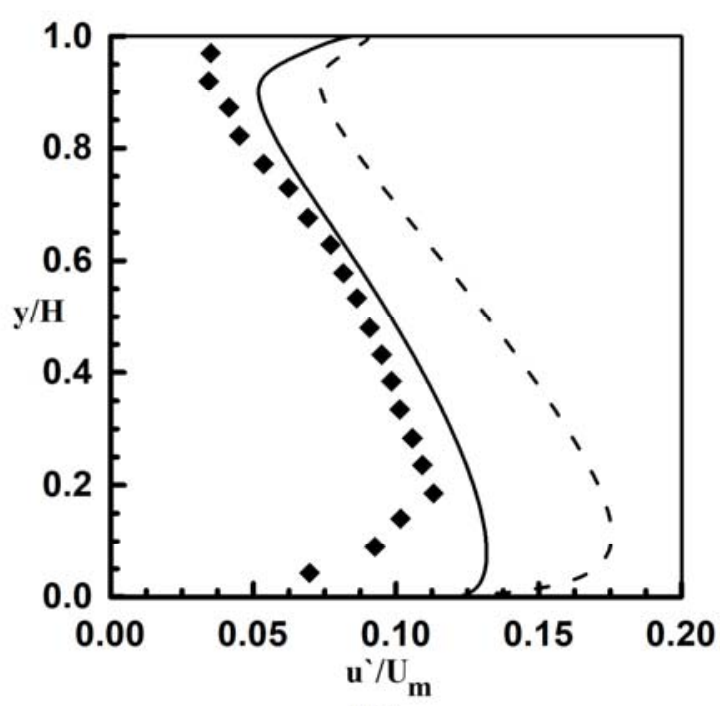

c. $x=450 \mathrm{~mm}$

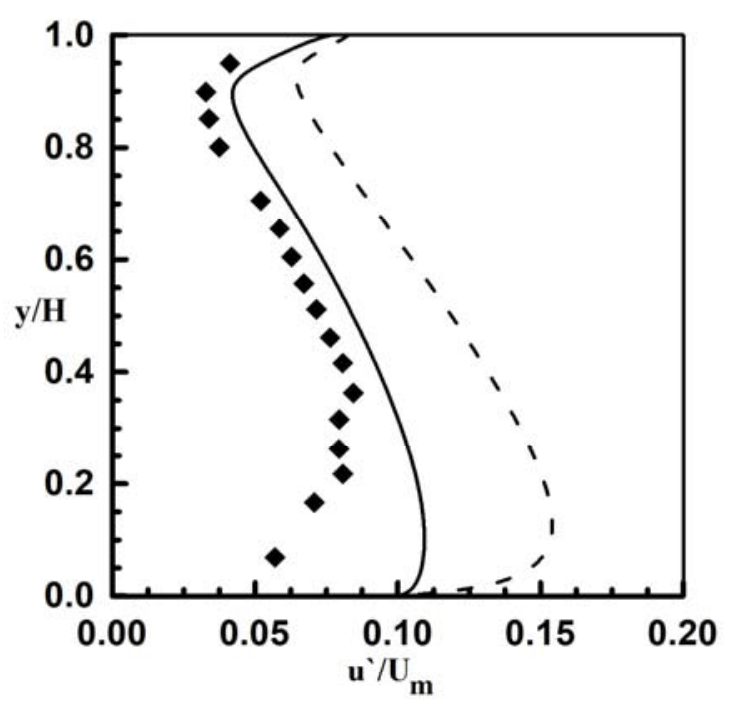

b. $x=350 \mathrm{~mm}$

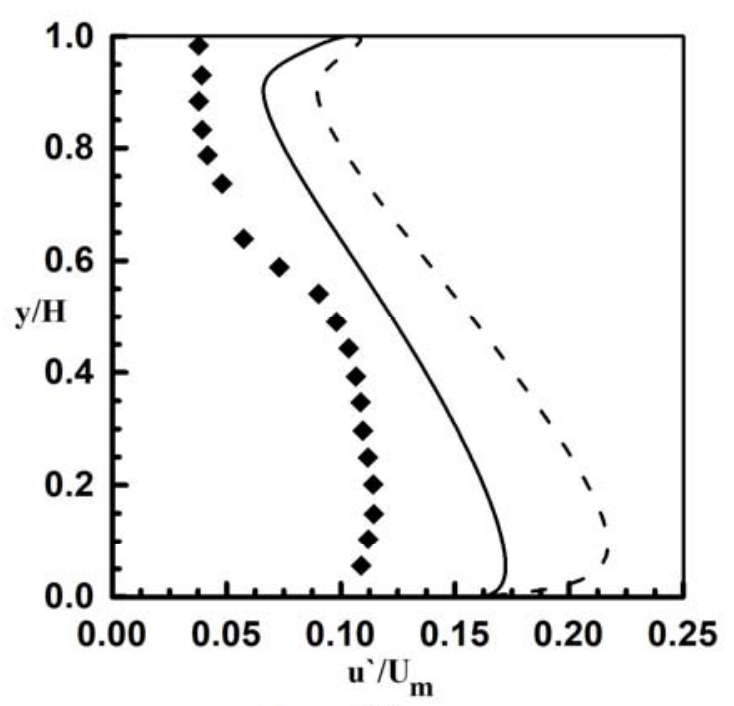

d. $x=570 \mathrm{~mm}$

Figure 4. Streamwise root-mean-square (RMS) turbulence intensity at different locations 


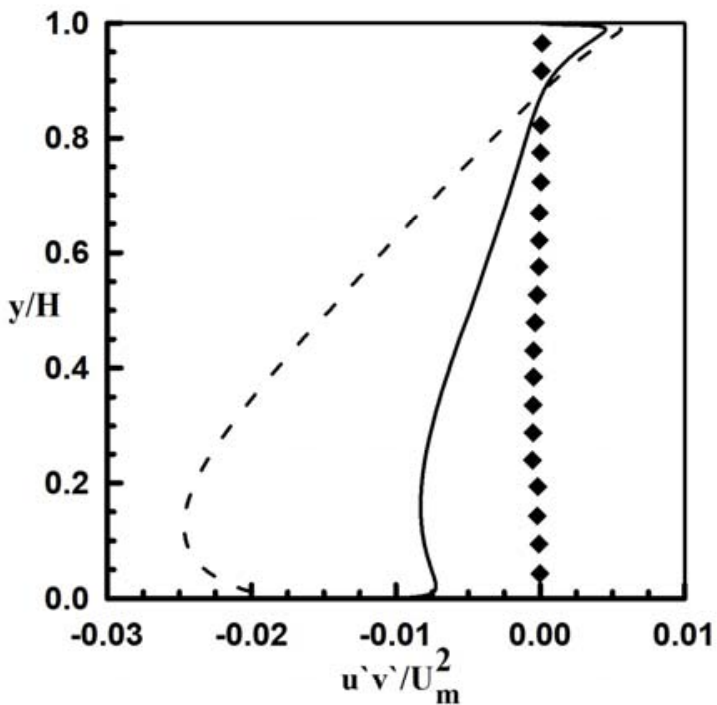

a. $x=220 \mathrm{~mm}$

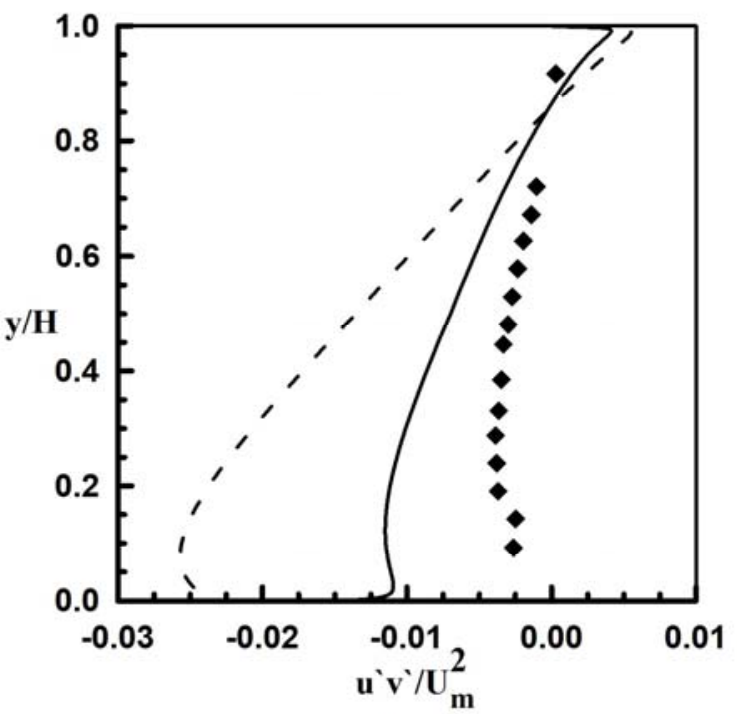

c. $x=450 \mathrm{~mm}$

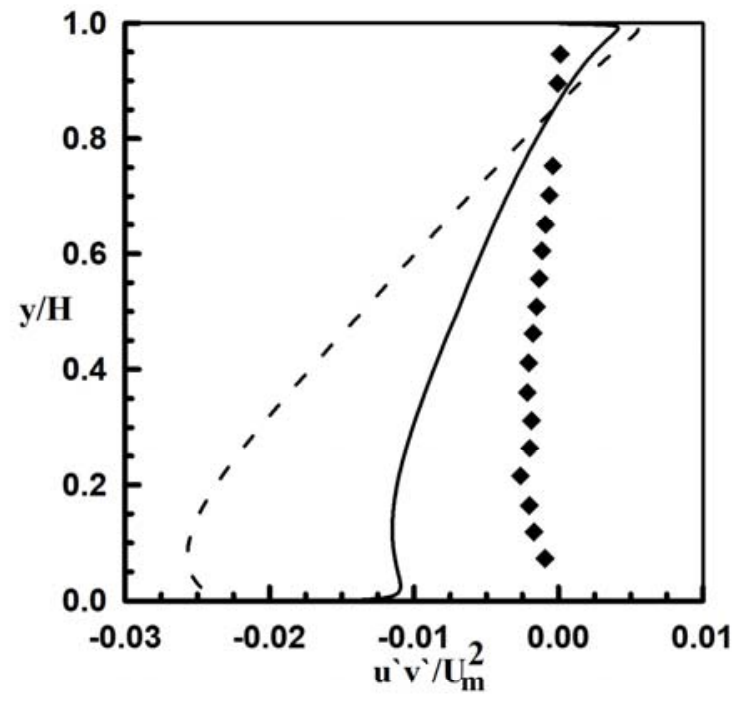

b. $x=350 \mathrm{~mm}$

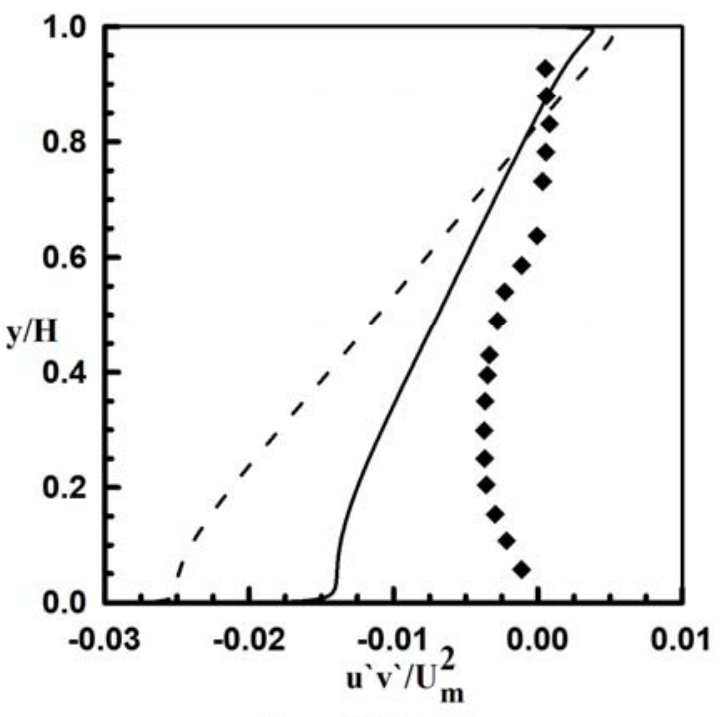

d. $x=570 \mathrm{~mm}$

Figure 5. Turbulent shear stress at different locations

\subsection{Case III}

The third set of the results include both the endwall disturbance and the sidewall injection. The numerical simulation is performed in case of laminar regime (with out including any turbulence model) and in case of turbulence modelling via the CH-TM [7]. Moreover, the coupling between the induced acoustics and the turbulence intensity is considered. Figure 6 shows comparison of axial velocity and pressure time histories for lamianr and turbulent flow. The left hand side represents the velocity time history, while the pressure time illustrated on the right side. It is clearly noticed that the deviation between the laminar and turbulent solution for the velocity and pressure time history has a significant change as axial distance increasing downstream. 
Near the aft end, the velocity time histories show very small deviation between the laminar and turbulent flow, while the pressure time history reveals a fairly significant deviation between the two solutions. This trend may be related to the complex acoustic wave patten mechanism and the turbulence intensity values everywhere in the chamber. Our previous results in [4] showed that the latter one attains maximum values near the exit plane for the steady state solution and the effect of turbulence is clearly seen after the third-fourth of the chamber near the exit as shown in Fig 7. The surface profiles for the unsteady vorticity at different time for the laminar and turbulent flow is presented in Fig. 8.
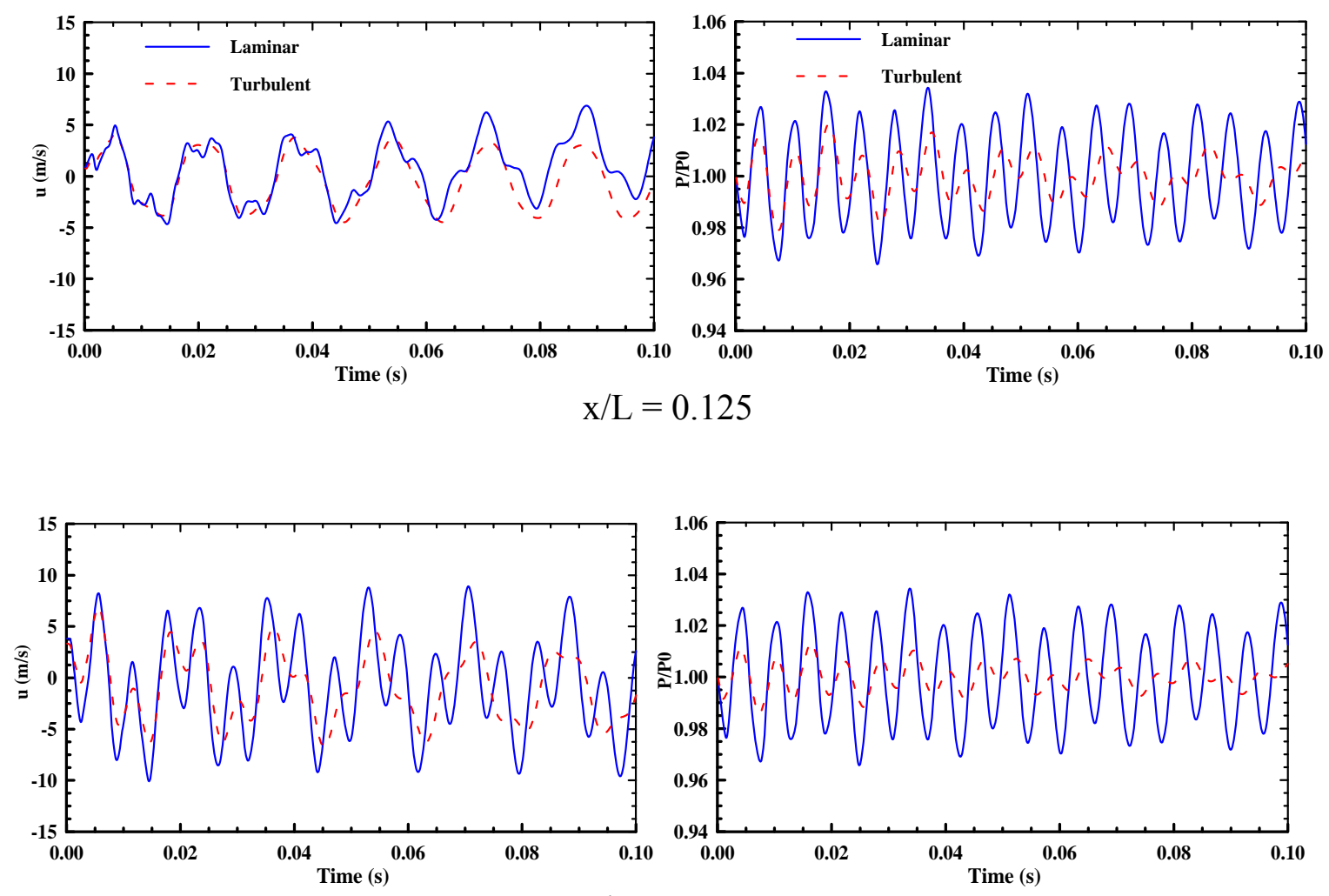

$\mathrm{x} / \mathrm{L}=0.557$
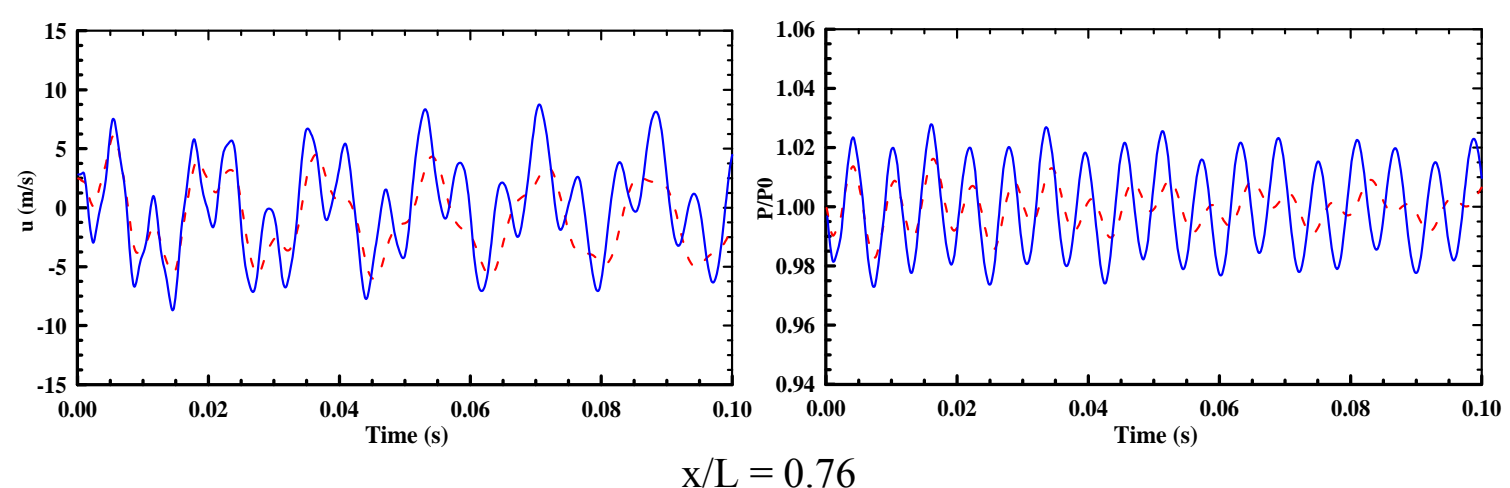

Figure 6. Comparison of axial velocity and pressure time histories for CASE III. 


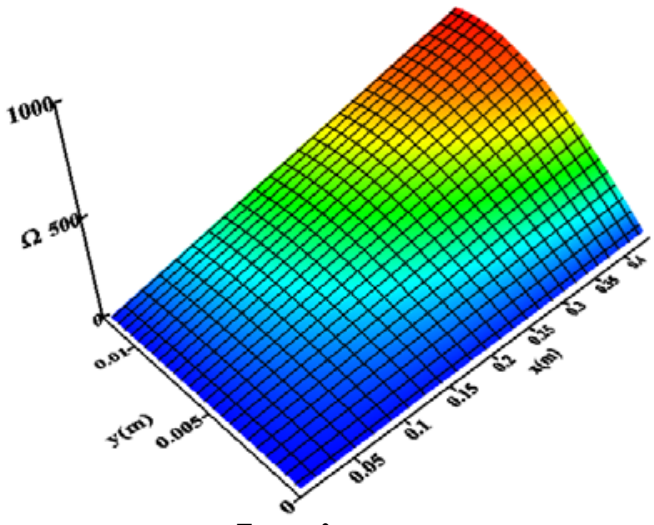

a. Laminar

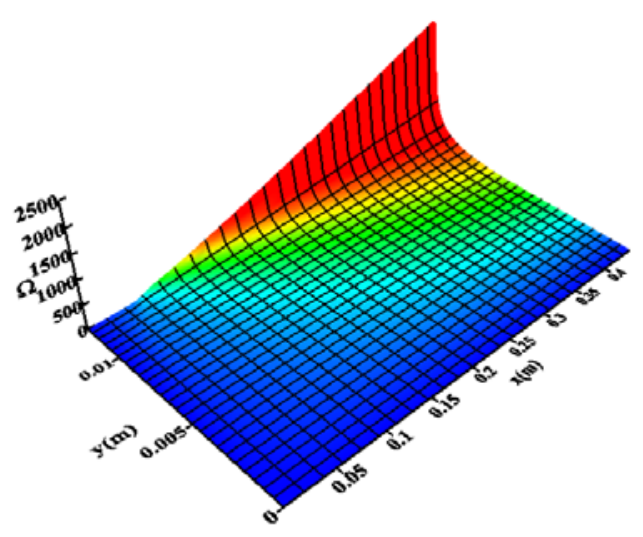

b. Turbulent

Figure 7. Steady state vorticity for laminar and turbulent solutions
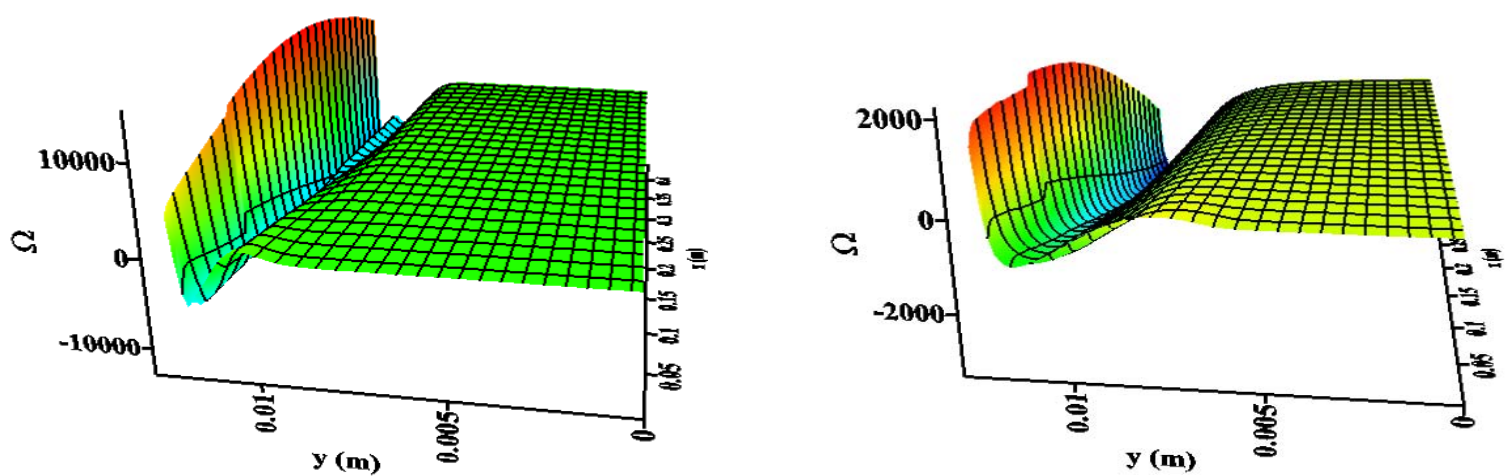

$\mathrm{t}=0.016 \mathrm{sec}$
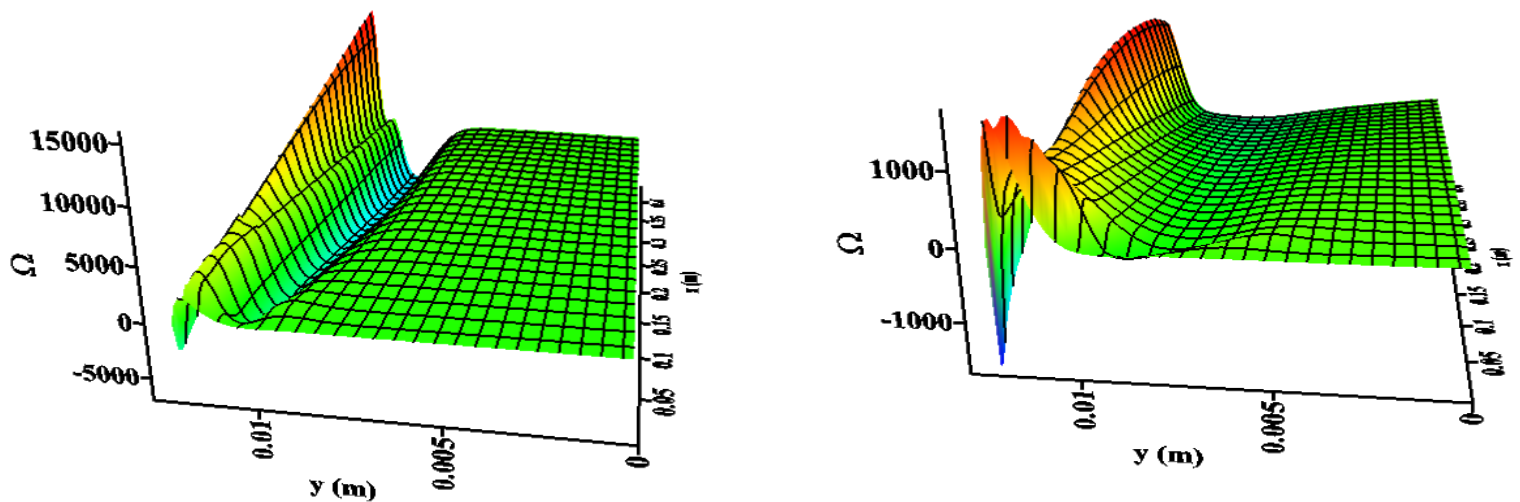

$\mathrm{t}=0.024 \mathrm{sec}$
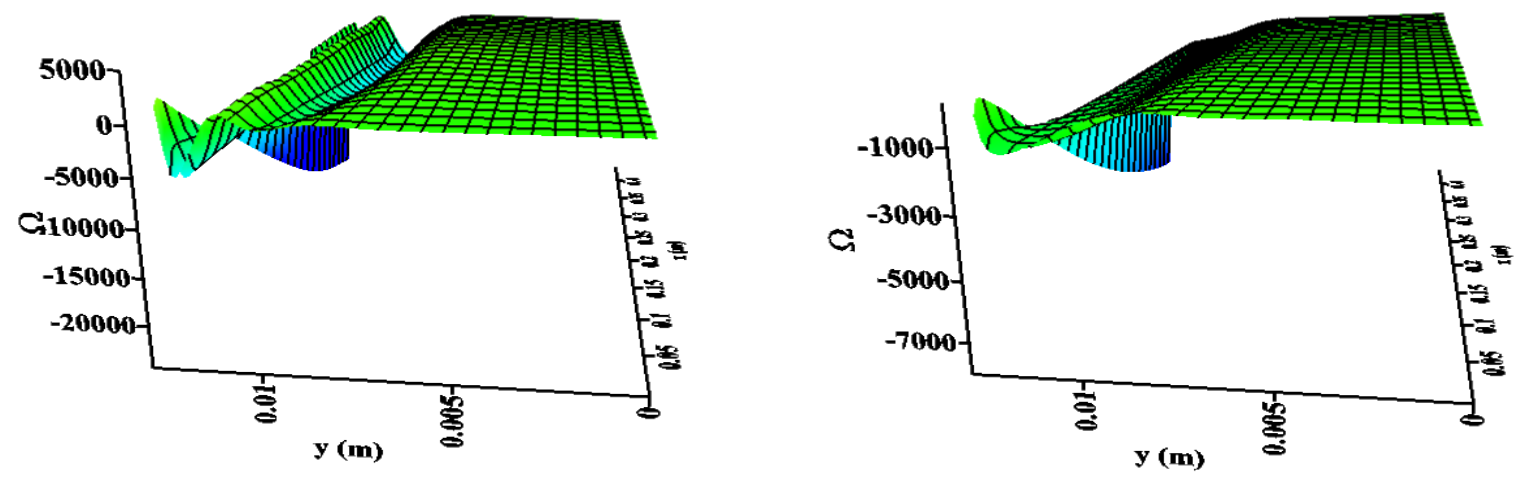

$\mathrm{t}=0.032 \mathrm{sec}$

Figure 8. Unsteady vorticity for laminar and turbulent solutions: Lift column represents laminar flow and right column refers to turbulent flow 


\section{Conclusion}

The internal flow field in a solid rocket motor chamber SRMC with compressible transpiration and endwall disturbance is numerically investigated. Three different cases are considered. The first case of the study is directed to pure acoustic fields due to endwall disturbance, while the second one considered steady state rotational flow due to steady sidewall injection. The last set of the results is performed to account the complete acousticfluid dynamics interaction generated from an endwall disturbance and sidewall injection.

The numerical results are validated firstly through a comparison with the analytical analysis and the available experimental measurements for the first and the second case, respectively. The comparisons showed a general fairly agreement for the axial velocity and pressure profiles at different locations in SRMC. The effect of including the nonlinear terms could be visibly observed in predicting higher harmonics oscillation in the numerical results rather the analytical ones. The second case comparison showed the effectiveness of the adopted turbulence models although of its simplicity and computational economics. In the third case, the effect of including turbulence models in predicting the internal flow characteristics is explained by a comparison between the laminar and the turbulent simulation.

The comparison showed for the first time the coupling between the generated unsteady vorticity arising from acoustics-fluid dynamics interaction and the turbulence intensity that exist everywhere in the chamber. The former one is studied extensively by Hegab and Kassoy [22] and showed how the acoustic-fluid interaction might impact the burning rate and, in turn, had a great effect on the erosive burning. Here in the current study, there is a novel essential coupling by considering the turbulence exist accompany to acoustic-fluid dynamics interaction. This essential coupling may add another factor that controls the erosive burning in real solid rocket motor chamber. In general, more intensive computational work is needed to extend the current research to account for the combustion of real solid rocket motor propellant.

\section{Acknowledgment}

This work is supported by the Science and Technology Development Fund (STDF) through the project ID-108.

\section{References}

[1]. Wang, M. and Kassoy, D. R., Nonlinear oscillations in a resonant gas column: An initial-boundary-value study, SIAM, J. App. Math., 55 (4), 923-951, 1995.

[2]. Hegab, A. M., Vorticity generation and acoustic resonance of simulated solid rocket motor chamber with high wave number wall injection, Computers \& Fluids 38, 12581269, 2009.

[3]. Apte, S.V. and Yang, V., Unsteady flow evolution in porous chamber with surface mass injection, Part 1: Free oscillation, AIAA, 39(8), 244-253, 2001.

[4]. El-askary, W. A., Balabel, A., El-behery, S. M. and Hegab, A., Prediction of Turbulence Transpiration Resulting from Asymmetric and Symmetric Mass Injection in a Porous Channel, Proceeding of ICFD10, Ain Soukhna, Egypt, Dec. 16-19, 2010.

[5]. Balabel, A., Hegab, A. M., Nasr, M. and El-Behery, S. M., Assessment of turbulence modeling for gas flow in two-dimensional convergent-divergent rocket nozzle, Applied Mathematical Modelling, 35, 3408-3422, 2011. 
[6]. Launder, B. E.and Spalding, D. B., The numerical computation of turbulent flows", Computer Methods in Applied Mechanics and Engineering, 3, 269-289, 1974.

[7]. Chen, Y.S. and. Kim, S.W., Computation of turbulent flows using an extended $k-\varepsilon$ turbulence closure model", NACA-CR-179204, 1987.

[8]. Apte, S.V. and Yang, V., A large-eddy simulation study of transition and flow instability in a porous-walled chamber with mass injection, J. Fluid Mech., 477, 215225, 2003.

[9]. Rhea, S., Bini, M., Fairweather, M. and Jones, W.P., RANS modelling and LES of a single-phase, impinging plane jet, Computers and Chemical Engineering 33, 1344 1353, 2009.

[10]. Zhao Q, Staab PL, Kassoy DR, Kirkkopru K., Acoustically generated vorticity in an internal flow. J. Fluid Mech., 13, 247-85, 2000.

[11]. Wang, M. and Kassoy, D. R., Nonlinear oscillations in a resonant gas column: An initial-boundary-value study, SIAM, J. App. Math., 55 (4), 923-951, 1995.

[12]. Sileem, A. and Nasr, M., An experimental study on finite amplitude oscillations in ducts: the effect of adding variable area part to the open end of constant-area resonant tube, Alexandria Engineering Journal, 42(4), 397-409, 2003.

[13]. Nasr, M., Hegab, A. M., El-Askary, W. A. and Yousif, K. A., An Investigation on the Internal Flow in Simulated Solid Rocket Motor Chamber/Nozzle Configuration, $13^{\text {th }}$ International Conference on AEROSPACE SCIENCES \& AVIATION TECHNOLOGY, ASAT- 13, Cairo, Egypt, May 26 - 28, 2009.

[14]. Hinze, J. O., Turbulence, McGraw-Hill Publishing Co., New York, 1975.

[15]. Patankar, S.V., "Numerical heat transfer and fluid flow", McGraw-Hill, New York, USA, 1980.

[16]. Karki, K.C., "A calculation procedure for viscous flows at all speeds in complex geometries", Ph.D. Thesis, University of Minnesota, 1986.

[17]. Avalon, G., Casalis, G., and Griffond, J. 1998 Flow instability and acoustic resonance of channels with wall injection. AIAA Paper 98-3218.

[18]. Deng, Z., Addrian, R.J and Tomkinson, C.D.," Structure of Turbulence in Channel flow with a Fully Transpired Wall", AIAA paper 2001-1019, 39 ${ }^{\text {th }}$ Aerospace Sciences Meeting and Exhibit, Jan. 8-11, Reno, NV, USA.

[19]. Hegab, A.M. and Nasr, M., "Unsteady Vorticity Fields in A long Narrow Channel with Endwall Disturbances and Sidewall Injection", EG-220, $9^{\text {th }}$ International Congress of Fluid Dynamics \& Propulsion Alexandria, Egypt, Dec. 18-21, 2009.

[20]. Kirkkopru, K., Kassoy, D.R. and Zhao, Q., "Unsteady Vorticity Generation and Evaluation in a Model of Solid Rocket Motor", J. of Propulsion and Power, Vol. 12, No.4, 1996, pp. 646-654.

[21]. Balabel, A. and El-Askary, W., On the performance of linear and nonlinear $\mathrm{k}-\varepsilon$ turbulence models in various jet flow applications, European Journal of Mechanics B/Fluids, 30 (2011) 325-340.

[22]. Hegab, A.M., and Kassoy, D.R., (2006) "Internal Flow Temperature and Vorticity Dynamics Due to Transient Mass Addition", AIAA Journal, Vol.44, No.4, April 2006. 